\title{
Fungal Contaminants in Cytopathology Specimens
}

\author{
Prashant Sharma ${ }^{1}$, Neeta Kumar², Shyama Jain ${ }^{3}$
}

A pseudo-epidemic of environmental fungi, most likely by Fusarium spp., leading to inappropriate investigations for disseminated systemic mycosis is described. Subtle diagnostic clues, including the specimens affected, the nature of the host response, and the type of fungal elements noted helped to determine the nature of contaminants. The potential pitfall can be avoided by the knowledge of pertinent disease biology, prompt consultation for infectious diseases, and investigations of the potential environmental sources followed by source control. (Biomed J 2014;37:31-32)

\section{Key words: cytological examination, fungal infection, fusariosis, immunocompromised state, neutropenia, smear contaminants}

$\mathrm{W}$ e would like to share our experience in the artifacts on cytological smears that led to unwarranted investigations in a case. Fine needle aspiration from an enlarged cervical lymph node of a febrile neutropenic 4-year-old boy with relapsed acute lymphoblastic leukemia showed fungal macroconidia in a necrotic background [Figure 1]. Since the widely distributed filamentous fungi commonly found in soil and plants, such as Fusarium and Aspergillus spp., are known to cause systemic infections in immunocompromised hosts, ${ }^{[1,2]}$ and the only inflammatory response in neutropenic patients may be necrosis, a medical microbiology opinion was sought on the nature of the fungal elements.

However, in the next 2 days, 13 other fine needle aspirates processed in the same laboratory and from different anatomic sites in unrelated immunocompetent patients also revealed macroconidia. Neither hyphal forms nor host responses were observed in all cases, and none of the cases had any risk factors for disseminated fungemia. Moreover, other pathological findings that accounted for the clinical observations were present. The macroconidia was thus determined to be caused by the contaminants. The microbiologist also commented that adventitial conidiogenesis in the absence of hyphae is not seen in human fusariosis, and that the infections in the absence of obvious angioinvasion and thrombosis would be rare even in a neutropenic patient. ${ }^{[1,2]}$ With cultures, serology, and molecular investigations being already initiated in the index case based on verbal communication, all returned negative.
The environmental source of the contamination was traced to a broken window facing a hospital park in the laboratory. Fungal elements had most likely settled on the air-dried, unstained smears. This was corroborated by the fact that only the Giemsa- and Ziehl Neelsen-stained smears (that are air-dried) showed extraneous elements, whereas the Papanicoloau-stained ones (immersed immediately in fixative) did not. In addition, fixing the broken window solved the problem.

We report this "pseudo-outbreak" to remind the readers of the Biomedical Journal that it can be difficult to distinguish true but rare infections from the cases where pathological specimens were contaminated by other biological agents. ${ }^{[3-5]}$ The diagnostic pitfall can be avoided by the knowledge of pertinent disease biology, prompt consultation for infectious diseases, and investigations of the potential environmental sources followed by source control.

\section{Acknowledgment}

Each author has participated sufficiently in the work to take public responsibility for appropriate portions of the content.

\section{REFERENCES}

1. Boutati EI, Anaissie EJ. Fusarium, a significant emerging pathogen in patients with hematologic malignancy: Ten years' experience at a cancer center and implications for management. Blood 1997;90:999-1008.

From the ${ }^{1}$ Department of Haematology, Postgraduate Institute of Medical Education and Research, Chandigarh, India; ${ }^{2}$ Department of Pathology, Faculty of Dentistry, Jamia Milia Islamia University, New Delhi, India; ${ }^{3}$ Department of Pathology (Cytopathology Laboratory), Maulana Azad Medical College and Lok Nayak Hospital, BSZ Marg, New Delhi, India

Received: Jan. 15, 2013; Accepted: Aug. 05, 2013

Correspondence to: Dr. Prashant Sharma, Department of Haematology, Postgraduate Institute of Medical Education and Research, India. Sector 12, Chandigarh - 160 012, India. Tel: 91-8872016123; Fax: 91-1722744401; E-mail: sharma.prashant@ pgimer.edu.in

DOI: $10.4103 / 2319-4170.117898$ 


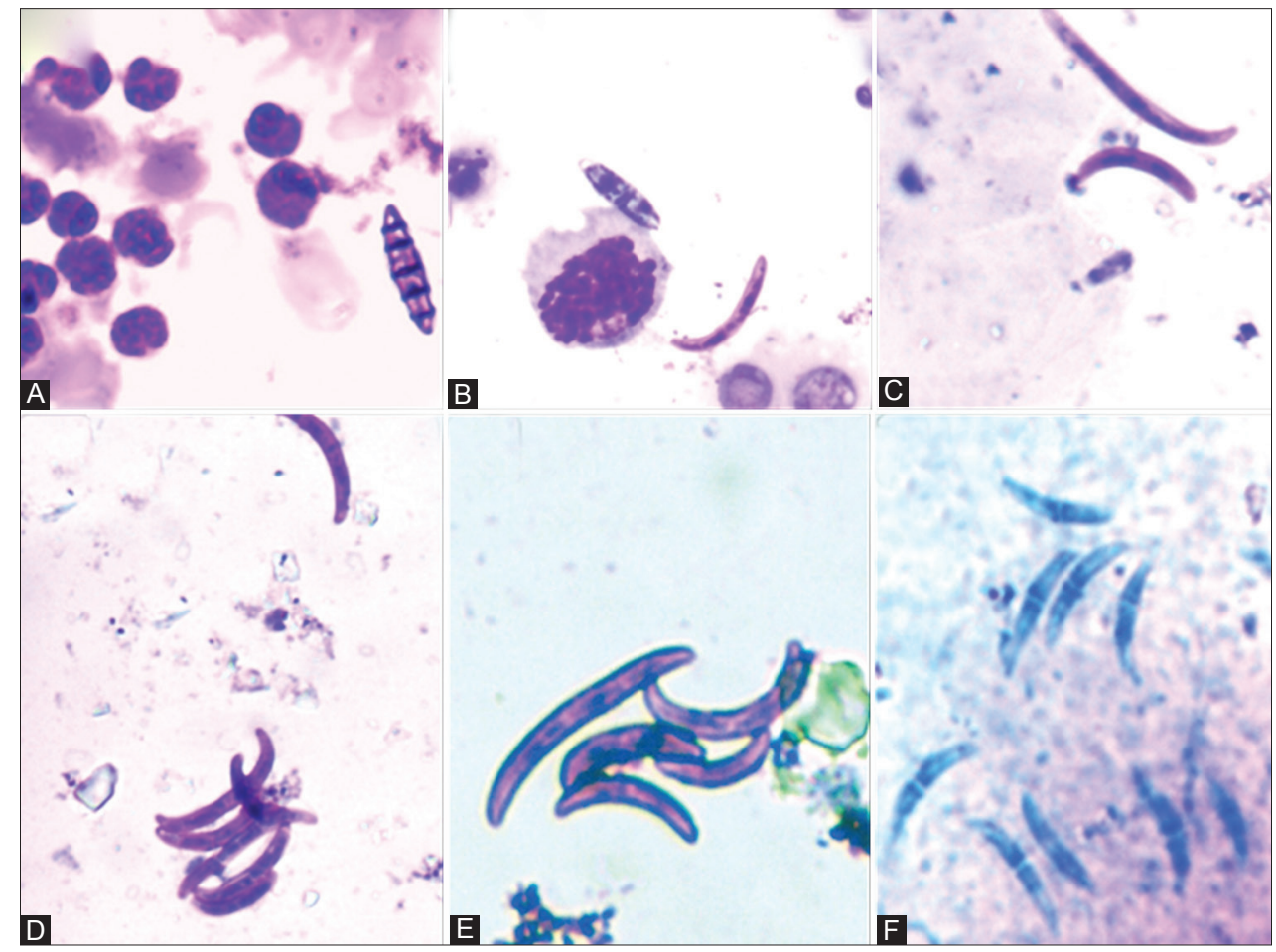

Figure 1: Fusarium macroconidia from various specimens. A and B are fine needle aspirates from a cervical lymph node in a child with relapsed acute lymphoblastic leukemia. $\mathrm{C}$ and $\mathrm{D}$ are from an aspirate of a thyroid nodule showing colloid goiter. $\mathrm{E}$ is from a subcutaneous lipoma, while F is from a purulent aspirate from an abscess. Smears A-E were stained by Giemsa stain $(\times 400)$, while F was stained by Ziehl Neelsen stain for acid-fast bacilli with methylene blue counterstain $(\times 400)$.

2. Reiss E, Shadomy HJ, Lyon GM. Fundamental Medical Mycology. New York: Wiley Blackwell; 2011.

3. Grigis A, Farina C, Symoens F, Nolard N, Goglio A. Nosocomial pseudo-outbreak of Fusarium verticillioides associated with sterile plastic containers. Infect Control Hosp Epidemiol 2000;21:50-2.

4. O’Donnell K, Sutton DA, Rinaldi MG, Magnon KC, Cox PA,
Revankar SG, et al. Genetic diversity of human pathogenic members of the Fusarium oxysporum complex inferred from multilocus DNA sequence data and amplified fragment length polymorphism analyses: Evidence for the recent dispersion of a geographically widespread clonal lineage and nosocomial origin. J Clin Microbiol 2004;42:5109-20.

5. Jain S, Sharma P, Gupta R. Dual cervical parasitosis in a psychiatric patient. Cytopathology 2005;16:53-4. 